\title{
Tuberculous Abscess of the Psoas Muscle in a Patient with Acute Lumbar Burst Fracture: A Missed Diagnosis
}

\author{
Jin Sung Cheong', Ki Seong Eom ${ }^{2}$ \\ ${ }^{1}$ Departments of Neurology, ${ }^{2}$ Neurosurgery, Wonkwang University School of Medicine, Iksan, Korea
}

The authors present a rare case of tuberculous spondylitis and a large abscess in the left psoas muscle that occurred after spinal surgery for an acute traumatic burst fracture of the $L 2$ vertebral body. We retrospectively reviewed the patient's first magnetic resonance imaging (MRI) we found that some unusual findings, indicative of psoas abscess had been overlooked. As a result, diagnosis and treatment of tuberculous psoas abscess and spondylitis were considerably delayed. Despite the critical condition of patients in a similar emergency, surgeons should always pay close attention to the radiological findings and clinical symptoms of the patient before considering a surgical intervention or biopsy.

Key Words: Tuberculosis · Psoas abscess $\cdot$ Spondylitis

\section{INTRODUCTION}

Psoas abscess is characterized by infrequent incidence, insidious onset, and nonspecific clinical features, which can lead to misinterpretation and delayed diagnosis; thus, it is difficult to clinically diagnose psoas abscess ${ }^{1,5)}$. Here, we present a rare case of tuberculous spondylitis and a large abscess in the left psoas muscle that occurred after spinal surgery for an acute burst fracture of the second lumbar (L2) vertebral body caused by a traffic accident. Moreover, the first radiological examination revealed some unusual findings that could have possibly indicated abscess before his spinal surgery.

\section{CASE REPORT}

In May 2009, a 44-year-old man was transferred to our hospital for lower back pain after he suffered from a blunt trauma in a pedestrian traffic accident. His medical and surgical histories were unremarkable. Neurological examination and chest radiology revealed no abnormalities. Laboratory evaluation revealed a erythrocyte sedimentation rate (ESR) of $48 \mathrm{~mm} /$ $\mathrm{hr}$ (normal range, $0-10 \mathrm{~mm} / \mathrm{hr}$ ) and c-reactive protein (CRP)

- Received: July 22, 2011 - Revised: December 20, 2011

- Accepted: January 2, 2012

Corresponding Author: Ki Seong Eom, MD, PhD

Department of Neurosurgery, Wonkwang University School of Medicine,

344-2 Shinyong-dong, Iksan 570-749, Korea

Tel: +82-63-859-1467, Fax: +82-63-852-2606

E-mail: kseom@wonkwang.ac.kr of $239.52 \mathrm{mg} / \mathrm{L}(0-5 \mathrm{mg} / \mathrm{L})$, and a normal WBC count of 7740 / $\mu \mathrm{l}(4000-10,000 / \mu \mathrm{l})$. Simple radiographs revealed a compression of L2 vertebral body. Lumbar computed tomography (CT) scan with bone window settings revealed a burst fracture of the L2 vertebral body with displacement of a posterior fragment into the spinal canal (Fig. 1A). Lumbar magnetic resonance imaging (MRI) revealed acute burst fracture with retropulsion of the posterior bone fragment at the L2 vertebral body and acute compression fracture of the L1 vertebral body (Fig. 1B). He underwent posterior reduction, transpedicular screw fixation, secondary anterior thoracoscopic decompression, and anterior reduction that was accomplished with a distractible cage and a modular anterior construct systemthoraco-lumbar (MACS-TL) plate (Aesculap, Tuttlingen, Germany) (Fig. 1C). The patient had no postoperative complications and was discharged on the $45^{\text {th }}$ postoperative day.

Until July 2010, the patient's clinical condition was satisfactory; thereafter, he exhibited a gradually enlarging swelling in the left flank, associated with pain extending up to the left thigh, and urinary incontinence. He did not have productive cough or any other pulmonary symptoms. His ESR was $120 \mathrm{~mm} / \mathrm{h}$; CRP level, $59.94 \mathrm{mg} / \mathrm{L}$; blood urea nitrogen (BUN) level, $28.1 \mathrm{mg} / \mathrm{dL}$; and serum creatinine level, $1.77 \mathrm{mg} / \mathrm{dL}$. Abdominopelvic CT revealed the presence of a large abscess in the following sites: left psoas muscle, lower pole of the left kidney, and posterolateral abdominal wall. Communication at all abscess sites was noted (Fig. 2). Abscess drainage and irrigation and partial nephrectomy through the previous surgical route were performed under general anesthesia. The abscess and surrounding soft tissue samples yielded positive results 

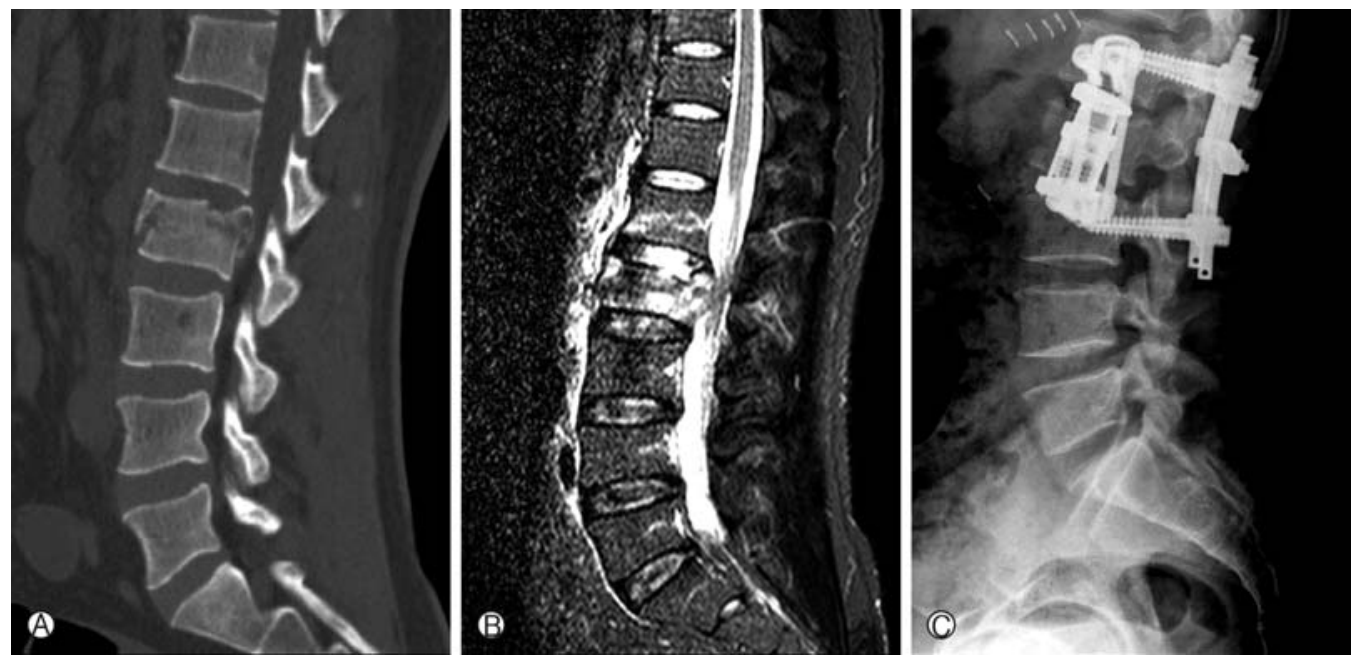

Fig. 1. (A) Preoperative sagittal lumbar computed tomography scan with bone window settings showing burst fracture of the $L 2$ vertebral body with displacement of a posterior fragment into the spinal canal. (B) Preoperative sagittal lumbar STIR magnetic resonance imaging showing acute burst fracture with retropulsion of the posterior bone fragment at the L2 vertebral body and acute compression fracture of the L1 vertebral body. (C) Postoperative lateral radiograph of the lumbar spine showing posterior fixation with pedicular screws and anterior reduction accomplished with a distractible cage and a MACS-TL plate (Aesculap, Tuttlingen, Germany).
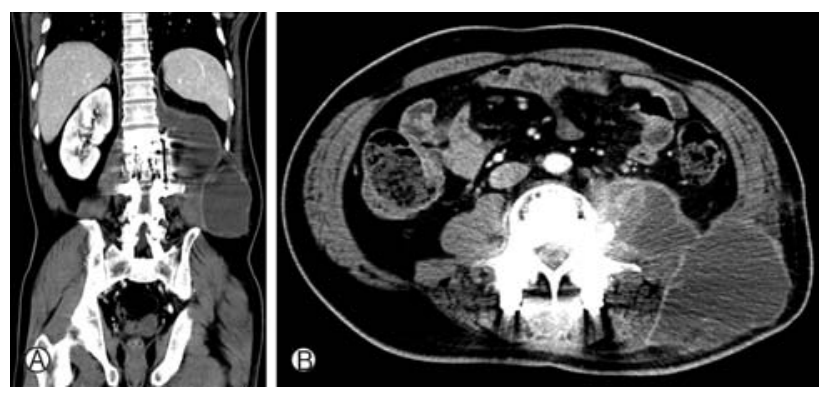

Fig. 2. Coronal (A) and axial (B) abdominopelvic computed tomography scan showing the presence of a large abscess in the left psoas muscle, lower pole of the left kidney, and posteriolateral abdominal wall. Communication at all abscess sites can be seen.

in the acid-fast bacilli (AFB) culture and polymerase chain reaction (PCR) for Mycobacterium tuberculosis complex. Histological examination revealed chronic granulomatous inflammation with necrosis. We retrospectively reviewed his first MRI taken prior to the spinal surgery. We realized that we had overlooked some unusual findings (described in Discussion). The patient received antituberculous chemotherapy, consisting of isoniazid, rifampin, pyrazinamide, along with pyridoxine supplements, on a daily basis. In November 2010, he again underwent abscess drainage and irrigation for abscess recurrence and wound dehiscence. The patient was transferred to the urological department for treatment of the left ureteral stricture.

\section{DISCUSSION}

The etiology of psoas abscess has been constantly changing during the last century. Initially, the predominant pathogen was Mycobacterium tuberculosis, which caused psoas abscess through either hematogenous spread or direct extension from vertebral osteomyelitis of the lumbar spine (Pott's disease). With the decline of Mycobacterium tuberculosis as a major pathogen of psoas abscess in developed countries, the condition was mostly detected secondary to diseases of the digestive tract, with other pathogens such as Staphylococcus aureus gaining more dominance in the pathogenesis of this psoas ab$\operatorname{scess}^{4,7}$. However, in recent years, tuberculosis-induced psoas infections are again emerging with the recurrence of tuberculosis in industrial countries and increase in the number of human immunodeficiency virus (HIV) infections ${ }^{5}$. Mückley et $\mathrm{al}^{5)}$ emphasized the role of the spine as the primary source of infection for secondary psoas abscess. According to the most case reports on psoas abscesses, this condition is noted after spinal surgery or infection ${ }^{3,6}$.

Psoas abscess was a typically well-recognized complication of spinal tuberculosis ${ }^{2)}$. Tuberculous psoas abscess occurring secondary to direct extension from other adjacent structures or even hematogenous seeding from a distant site is less frequent $^{8)}$. However, there have been hardly any reports on tuberculous psoas abscess presenting as a manifestation of tuber- 

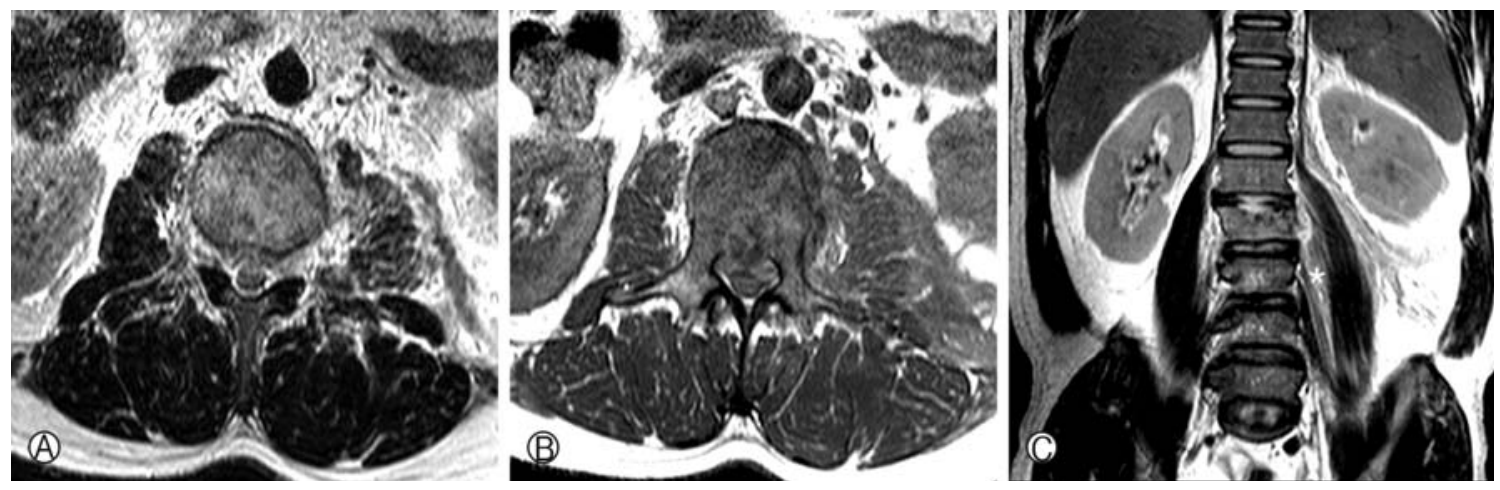

Fig. 3. (A, B) Preoperative axial magnetic resonance imaging (MRI) of the $L 2$ vertebra at the time of the first admission showing irregular shaped and ill-defined heterogeneously enlargement of the left psoas muscle compared to the right psoas or both paraspinal muscles. (C) Coronal T2-weighted MRI showing a thick line of high-signal intensity along the left psoas muscle (asterisk).

culosis, without evidence of an active infection elsewhere ${ }^{8)}$. Even in the case of our patient, there was no evidence of a tubercle bacillus infection anywhere but except in the surgical portion.

A retrospective review of the first MRI of our patient revealed some unusual findings of the left psoas muscle, which had been overlooked earlier. Axial MRI of the L2 vertebra revealed that the left psoas muscle was irregular shaped, ill-defined, and heterogeneously enlarged compared to the right psoas muscle and both paraspinal muscles at the level of the vertebral burst fracture. In addition, the coronal T2-weighted MRI revealed a thick line of high-signal intensity along the left psoas muscle (Fig. 3). Even though it is very difficult to determine whether asymmetric swelling of the left psoas muscle was due to trauma or infection, we consider that this unusual finding was a result of the infection. If it was caused by the trauma, there would have been more contusions of the paraspinal muscle than that of the psoas because the psoas is positioned anterior to the paraspinal muscle. Moreover, because the abnormality was detected only in the left psoas and not in either paraspinal muscle or the right psoas, it is difficult to determine whether the condition was a result of the trauma. We also consider that the possibility of tuberculous spondylitis extending to the vertebral body before injury is low. We assume that vertebral fracture and subsequent spinal surgery can make the progress of the primary psoas abscess around surrounding tissues quicker.

In the majority of earlier cases, the patients, as in our case, had presented with subacute or chronic symptoms and a good general state $^{8}$. Although fever, abdominal or back pain, and limited hip-joint mobility form the classical triad of psoas abscess, they can be inconsistently presented ${ }^{1)}$. Retrospective examination revealed that our patient also had abdominal pain and intermittent limited hip-joint mobility, weeks before the traffic accident. Therefore, suspicious clinical finding, radiological study, and microbial culture study of the pus, are crucial in the diagnosis of tuberculous psoas abscess.

\section{CONCLUSION}

This report might be significant, considering the possibility of missed diagnosis of psoas abscess in patients with acute lumbar compression fracture caused by trauma, even though the unusual findings of the left psoas muscle in the first MRI were not histologically examined. Unfortunately, because we mainly focused on the traumatic injury, we did not conduct a medical examination through interrogation and radiology prior to surgery, and as a result, diagnosis and treatment of psoas abscess and tuberculous spondylitis were considerably delayed. Therefore, despite the critical state of the patient in similar emergencies, surgeons should always pay close attention to the radiological findings and clinical symptoms of the patients, before considering a surgical intervention or biopsy.

\section{REFERENCES}

1. Chern $\mathrm{CH}$, Hu SC, Kao WF, Tsai J, Yen D, Lee CH: Psoas abscess: making an early diagnosis in the ED. Am J Emerg Med 15:83-88, 1997

2. Jung TG, Lee SH, Jang JS: Surgical Treatment of Thoracic Tuberculous Spondylitis Refractory to Anti-Tbc Medication. Korean J Spine 4:228-231, 2007

3. Korovessis P, Petsinis G, Papazisis Z: Unilateral psoas abscess following posterior transpedicular stabilization of the lumbar spine. Eur Spine J 9:588-590, 2000

4. Mallick IH, Thoufeeq MH, Rajendran TP: Iliopsoas abscesses. Postgrad Med J 80:459-462, 2004 
5. Műckley T, Schűtz T, Kirschner M, Potulski M, Hofmann G, B?hren V: Psoas abscess: the spine as a primary source of infection. Spine (Phila Pa 1976) 28:E106-113, 2003

6. Thomas E, Leroux JL, Segnarbieux F, Faure P, Bonnel F, Blotman F: Multiple psoas abscesses after posterior spinal fusion. Spine (Phila Pa 1976) 20:373-374, 1995
7. van den Berge M, de Marie S, Kuipers T, Jansz AR, Bravenboer B: Psoas abscess: report of a series and review of the literature. Neth J Med 63:413-416, 2005

8. Vaz AP, Gomes J, Esteves J, Carvalho A, Duarte R: A rare cause of lower abdominal and pelvic mass, primary tuberculous psoas abscess: a case report. Cases J 2:182, 2009 